\title{
Maximum Disparity Threshold Estimation for Stereo Imaging Systems via Variogram Analysis
}

\author{
Bogusław Cyganek and Jan Borgosz \\ University of Mining and Metallurgy \\ Department of Electronics \\ Al. Mickiewicza 30, 30-059 Kraków, Poland \\ \{cyganek, borgosz\}@uci.agh.edu.pl
}

\begin{abstract}
Estimation of the highest disparity value is very important for proper operation of any stereo matching algorithm. Choice of a too big or too small disparity range may cause severe errors in an output disparity map. A guess of this parameter by human is the method which is used very often during experiments, however it is not suitable in real time or autonomous vision systems. In the same way, a usage of the constant disparity range gives often poor results. The paper describes a innovative method of the automatic estimation of a maximum disparity for the real time stereo imaging systems. After short review of the most popular methods of the maximum disparity estimation, we present our method based on the variogram analysis. Finally the experimental results for the real images are also presented.
\end{abstract}

\section{Introduction}

During many experiments with the stereoscopic algorithms we have found, that right choice of the maximum disparity threshold value is crucial to the quality of the output disparity map and also influences the computation time [2] [4]. Choice of a too small value leads to false disparity map with many errors, whereas too big disparity range causes large errors as well, because of high amount of the false matches. Furthermore, selection of the disparity range should adapt to the true distance to the matched object. At the other hand, if we consider computation effort, then we obtain that smaller values for this parameter give shorter calculations time [1].

Having this in mind we have found that the estimation of the maximum disparity threshold is a critical problem for the real time stereo matching systems - for example a vision system which navigates blind person in real word. In this work we try to find solution of this problem. We rely on the statistic method - the variogram calculation and compare this approach to the ideal disparity estimation made by human [2]. 


\section{Review of the Methods of the Maximum Disparity Estimation}

In many stereo systems the disparity value was selected in the arbitrary way, due to the estimated geometry of the video acquisition system and the expected parameters of the analyzed scene. It is possible to rely on the theory which allows to limit top boundary of the disparity value to the difference between horizontal size of the picture and the current horizontal position (for a canonical stereo setup).

First solution to the estimation of the maximum disparity value were found to be an iteration method. This method uses fast but not very accurate matching algorithm to find the first estimate of the disparity range [2]. The subsequent stereo algorithm uses results of the first one. An another approach was proposed by Scharstein [6]. He suggest to cumulate gradient values of the quarter, then for the whole picture and based on this conjure the maximum disparity. Third solution is a scale-space approach [1][7] which allows to estimate disparity in different scales and then find the starting estimate. Described methods show that we can look for solution of the disparity range problem by performing the following operations on the picture:

- changing resolution of the picture and making disparity analysis in some regions,

- changing scale of the analysis,

- $\quad$ using more than one method on the unchanged picture.

Listed methods may be classified as a deterministic approaches. An opposite is a statistical approach which will be explored in this paper.

\section{Image Analysis by Variograms}

If we want to find a disparity between two images then we are interested in examining the spatial structure of a both images what can be stated in a form of an analysis of so called a regionalized variable [8]. One way to do this is to analytically relate the change of this variables as a function of some separating distance $h$. The solution of this problem may be in a form of a variogram, which defines a spatial correlation or structure of a regionalized function and is given by [5] [8]:

$$
\gamma(\mathrm{h})=\frac{1}{2} E\left([f(x)-f(x+h)]^{2}\right)=C(0)-C(h)
$$

where $C(h)$ is the covariance function, $E$ the mathematical expectation and $h$ the separating distance.

Shape of the variogram reflects the degree of correlation between samples of images. Variogram functions that rise as $h$ increases indicates that the spatial correlation decreases as more distant samples are chosen, until a separation distance is reached at which knowledge of one sample tells us nothing about another ones [ 3 ].

For two dimensional signals - such as images - the variogram was defined by Ramstein as follows: 
$\gamma(\mathrm{h})=\frac{1}{2 \mathrm{~N}_{\mathrm{c}} \mathrm{N}_{\mathrm{r}}} \cdot\left[\sum_{x=1}^{N_{c}} \sum_{y=1}^{N_{l}}[I(x+h, y)-I(x, y)]^{2}-\sum_{x=1}^{N_{c}} \sum_{y=1}^{N_{l}}[I(x, y+h)-I(x, y)]^{2}\right]$

where: $N_{c}$ and $N_{r}$ are respectively the number of rows and columns of the image, and $h$ is a distance expressed in pixels [[]].

In this paper we propose a modification to the equation (2) - we define variograms for the two images (stereo pair) as follows:

$$
\gamma(\mathrm{h})=\frac{1}{2 \mathrm{~N}_{\mathrm{c}} \mathrm{N}_{\mathrm{r}}} \sum_{x=1}^{N_{c}} \sum_{y=1}^{N_{r}}\left[I_{l}(x+h, y)-I_{r}(x, y)\right]^{2}
$$

where: $\mathrm{I}_{l}$ and $\mathrm{I}_{r}$ are the left and right pictures from the stereo pair, respectively.

\section{Disparity Range Estimation Based on Variogram Analysis}

As alluded to previously, in a case of the calculation of a variogram for two images (3), we expect to find correlation if pictures belong to a single stereo-pair. In other words, each displacement will increase a total decorrelation value between images.

Relying on this observation, after many experiments with variograms, authors have found some heuristic rule which allows to estimate maximum disparity value according to the following algorithm:

1) find value $h$ for which the variogram (3) reaches the first local minima,

2) increase $h$ about $10-20 \%$ to obtain an approximated value for a maximum disparity range of a given stereo-pair.

This heuristic rule may be justified by the observation that appearance of strong local minima corresponds to the high amount of points in a stereo pair with displacement equal to the argument $h$. It should be noticed that a value which was found with this method may not be a global maximum, because of the statistic nature of the calculations. However experimental results show that this method works in practice for most of real images.

\subsection{Experimental Results of the Heuristic Variogram Analysis}

Presented hereafter experiments are based on real stereo pairs depicted in Fig. 1. The images Fiesta, Phone and Street may be found as extremely difficult for matching, because of their content and imperfect way of the acquisition. The picture Trees is an interesting case due to very small disparity range.

In Fig. 2 we present variograms calculated for the tested stereo pairs. 

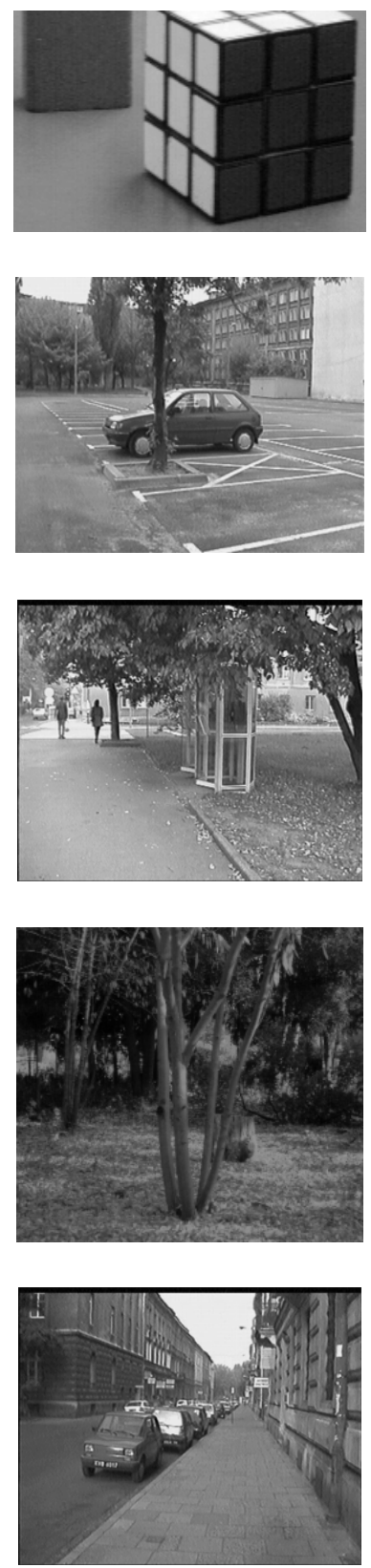

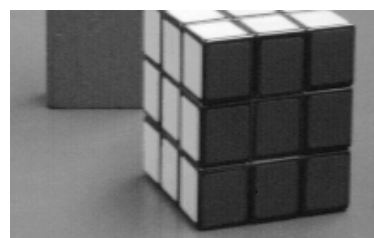

a

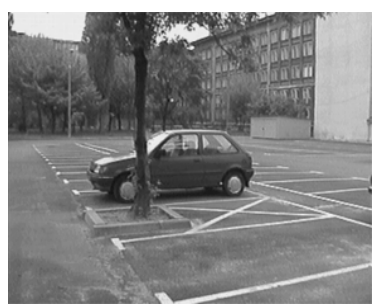

b

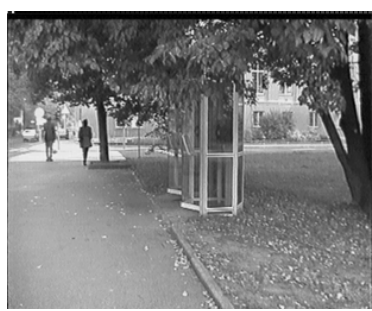

$\mathrm{c}$

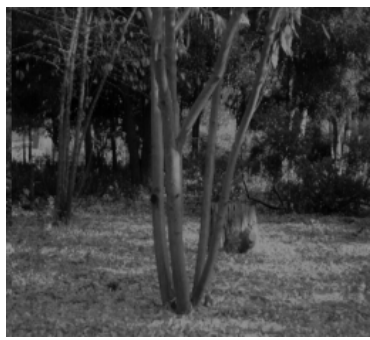

d

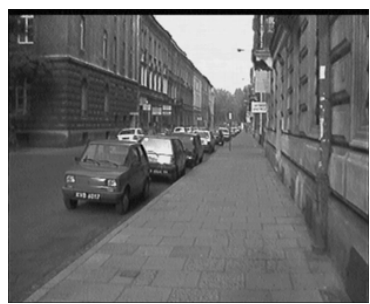

$\mathrm{e}$

Fig. 1. Stereo-pairs used during experiments: a) Rubic (CIL CMU), b) Fiesta (University of Mining and Metallurgy), c) Phone 2 (University of Mining and Metallurgy), d) Trees (SRI), e) Street 2 (University of Mining and Metallurgy) 


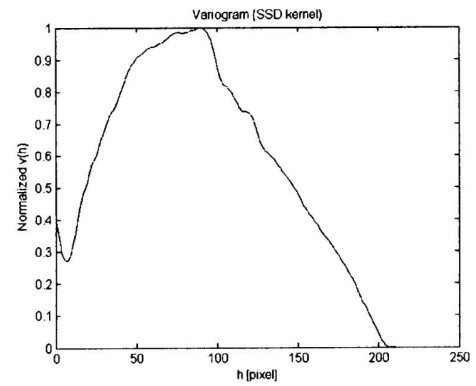

a

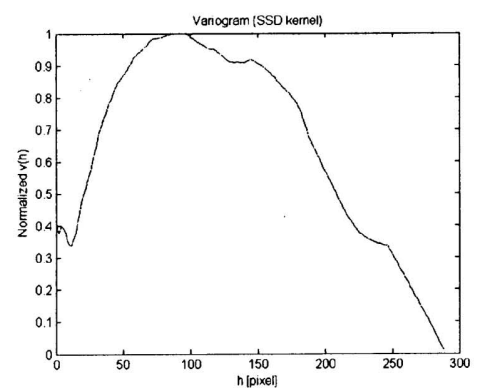

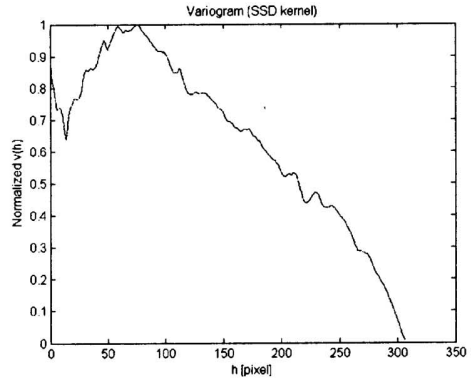

$\mathrm{b}$

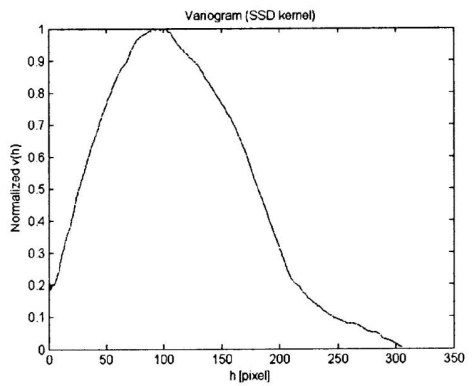

d

c

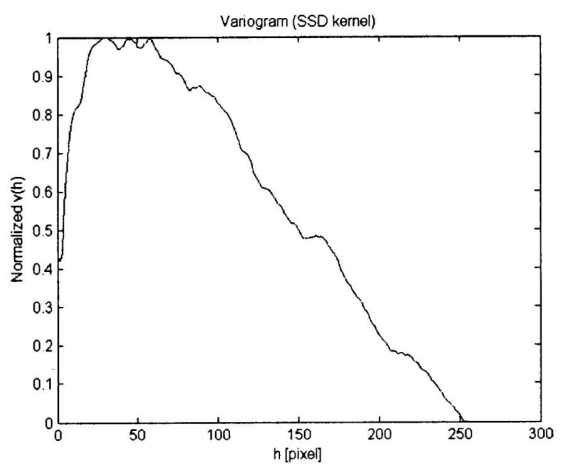

e

Fig. 2. Computed variograms for the tested stereopairs: a) Rubic, b) Phone 2, c) Fiesta, d) Street 2, e) Trees

For verification purposes, independently three persons with special software have estimated maximum disparity for the test pictures. Average values obtained by humans were compared with result obtained with the proposed method - comparison may be seen in Table 1. 
Table 1. A comparison of results obtained with the proposed method of a maximum disparity estimation and the estimate of this value made by persons

\begin{tabular}{|c|c|c|c|}
\hline Stereo pair & $\begin{array}{c}\text { Disparity range } \\
\text { estimated by human }\end{array}$ & $\begin{array}{c}\text { Disparity range } \\
\text { estimated with } \\
\text { presented method }\end{array}$ & Error \\
\hline Rubic & 11 & 8 & $37,5 \%$ \\
\hline Phone 2 & 21 & 16 & $31,25 \%$ \\
\hline Fiesta & 18 & 20 & $11,2 \%$ \\
\hline Street 2 & 17 & 16 & $6,25 \%$ \\
\hline Trees & 8 & 3 & $266,5 \%$ \\
\hline
\end{tabular}

\section{Modifications to the Variogram Analysis}

Obtained results are not satisfactory due to the obtained error range (see Table 1). We noticed that some improvements in our algorithm can be made by the following generalization of the variogram definition:

$$
\gamma_{\mathrm{K}}(\mathrm{h})=\frac{1}{2 \mathrm{~N}_{\mathrm{c}} \mathrm{N}_{\mathrm{r}}} \sum_{x=1}^{N_{c}} \sum_{y=1}^{N_{r}} K\left(I_{l}, I_{r}, x, y, h\right)
$$

where $K\left(I_{l}, I_{r}, x, y, h\right)$ is the kernel for the variogram. In the case of the classic definition (3), the varigram kernel is simply equal to the following:

$$
K\left(I_{l}, I_{r}, x, y, h\right)=\left[I_{l}(x+h, y)-I_{r}(x, y)\right]^{2}
$$

In the case of the variogram, it can be said, that for the given disparity value $h$, we perform operations defined by the kernel $K\left(I_{l}, I_{r}, x, y, h\right)$ in both images, and we sum and scale obtained result in the next step.

If we compare the Sum of Squared Differences metrics (SSD) given by the equation:

$$
M=\sum_{(x, y) \in U}\left(I_{1}(x, y)-I_{2}\left(x+d_{x}, y+d_{y}\right)\right)^{2}
$$

where $U$ denotes local neighborhoods and $d_{x}, d_{y}$ disparity values in both directions, than we will find that for the $d_{y}=0$ we obtain a classic variogram kernel given by (5).

By observed analogy we decided to make the analysis of the different kernels $K\left(I_{l}, I_{r}, x, y, h\right)$ forms based on the area-based stereo matching algorithms. We present all tested kernels in Table 2. 
Table 2. New variogram kernels. $S A D$ - Sum of Absolute Differences, ZSAD - zero mean $S A D, S S D$ - Sum of Squared Differences, ZSSD - zero mean SSD, SSD-N - SSD Normalized, $S C P$ - Sum of Cross Products, $S C P-N$ - Sum of Cross Products Normalized, CoVar Covariance-Variance

\begin{tabular}{|c|c|}
\hline Name & Kernel Formula $\boldsymbol{K}\left(\boldsymbol{I}_{\boldsymbol{p}} \boldsymbol{I}, \boldsymbol{x}, \boldsymbol{y}, \boldsymbol{h}\right)$ \\
\hline SAD & $\left|I_{1}(x, y)-I_{2}(x+h, y)\right|$ \\
\hline ZSAD & $\mid\left(I_{l}(x, y)-\overline{I_{l}}\right)-\left(I_{r}(x+h, y)-\overline{I_{r}}\right)$ \\
\hline$Z S S D$ & $\left(I_{l}(x, y)-I_{r}(x+h, y)\right)^{2}$ \\
\hline$S S D-N$ & $\left.\left[I_{l}(x, y)-\overline{I_{l}}\right]-\left[I_{r}(x+h, y)-\overline{I_{r}}\right]\right]^{2}$ \\
\hline$Z S S D$ & $\frac{\left[I_{1}(x, y)-I_{r}(x+h, y)\right]^{2}}{\sqrt{I_{l}(x, y)^{2} \cdot I_{r}(x+h, y)^{2}}}$ \\
\hline$S C P$ & $\frac{\left\lfloor\left(I_{l}(x, y)-\overline{I_{l}}\right)-\left(I_{r}(x+h, y)-\overline{I_{r}}\right)\right]^{2}}{\sqrt{\left(I_{l}(x, y)-\overline{I_{l}}\right)^{2} \cdot\left(I_{r}(x+h, y)-\overline{I_{r}}\right)^{2}}}$ \\
\hline$S C P-N$ & $\frac{I_{l}(x, y) \cdot I_{r}(x+h, y)}{I_{l}(x, y) \cdot I_{r}(x+h, y)}$ \\
\hline CoVar & $\frac{\sqrt{I_{l}(x, y)^{2} \cdot I_{r}(x+h, y)^{2}}}{\left.\sqrt{\left(I_{l}(x, y)-\overline{I_{l}}\right) \cdot\left(I_{r}(x+h, y)-\overline{I_{l}}\right)^{2} \cdot\left(I_{r}(x+h, y)-\overline{I_{r}}\right.}\right)^{2}}$ \\
\hline
\end{tabular}

All new variogram kernels were tested with the stereo pairs. Results of this tests for the exemplary Rubic stereo pair are presented in Fig. 3.

During experiments we have found that $S C P$ and $S C P-N$ kernels give output with falling characteristics. The $S C P$ measure is more interesting because of the lower requirements on a computation power.

\section{Disparity Range Estimation Based on the SCP Variogram}

Based on the observations about new variogram kernels we decided to use the $S C P$ kernel. After many experiments with this variogram, we have found next heuristic rule which allows to estimate maximum disparity value as follows:

- $\quad$ find the integer value $h$ which corresponds to the 0.94 value of the variogram

- $\quad$ take $h$ as the maximum disparity range.

This rule seems to be better than previous because:

- there is no need to look for the local minima,

- $\quad$ there is no need to make any modification of the $h$ value.

Results of the tests for the presented stereo-pairs are presented in the Fig. 4. Comparison with the true disparity ranges defined by the human is presented in Table 3. 


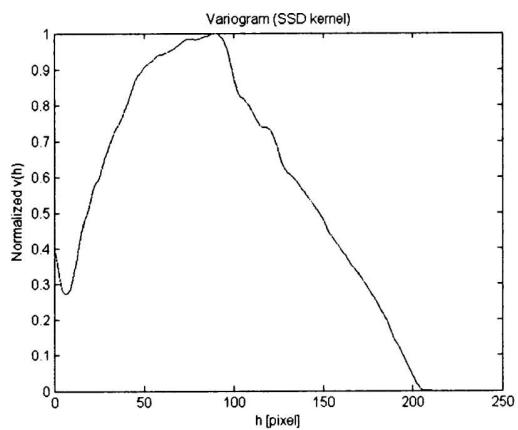

a

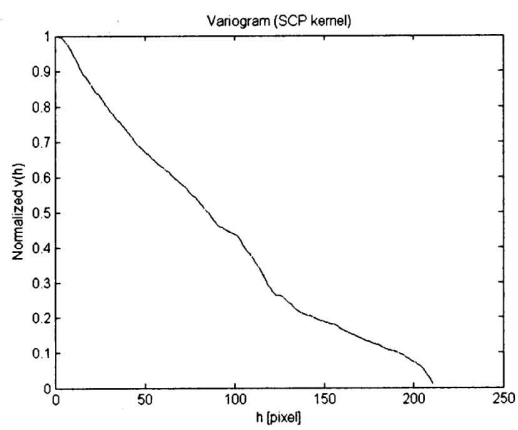

C

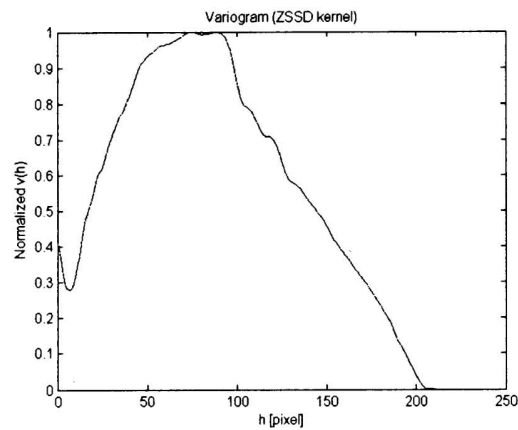

$\mathrm{e}$

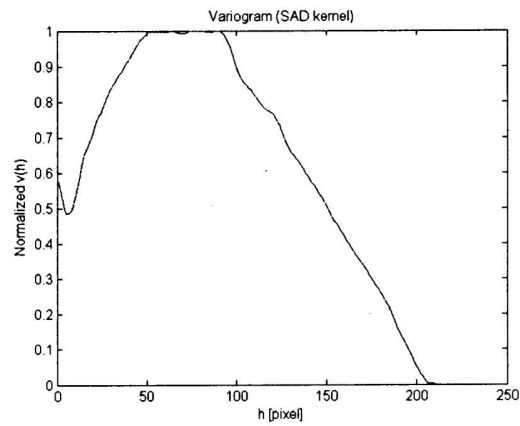

$\mathrm{b}$

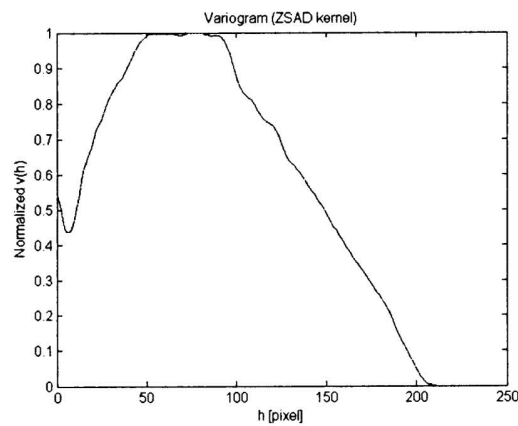

d

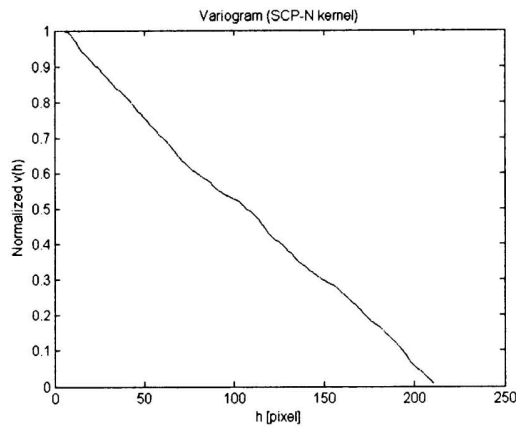

$\mathrm{f}$

Fig. 3. Examples of the new variogram kernels tested on the Rubic stereopair: a) SSD (classic), b) $S A D$, c) $S C P$, d) $Z S A D$, e) $Z S S D$, f) $S C P-N$ 


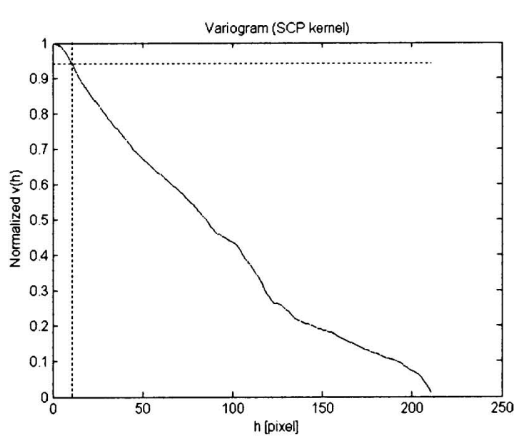

a

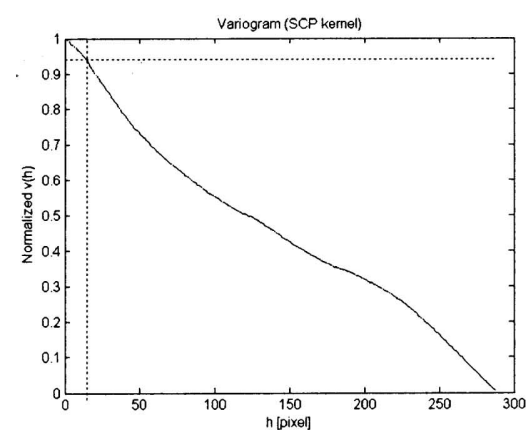

c

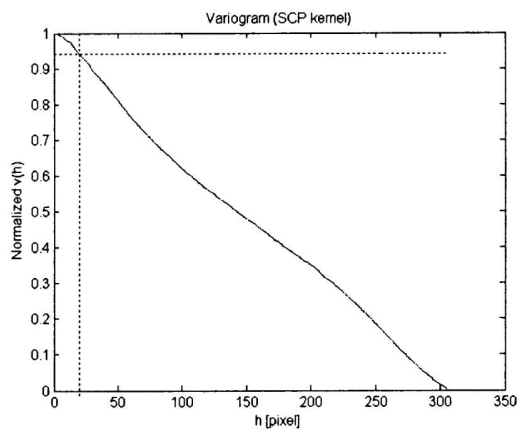

b

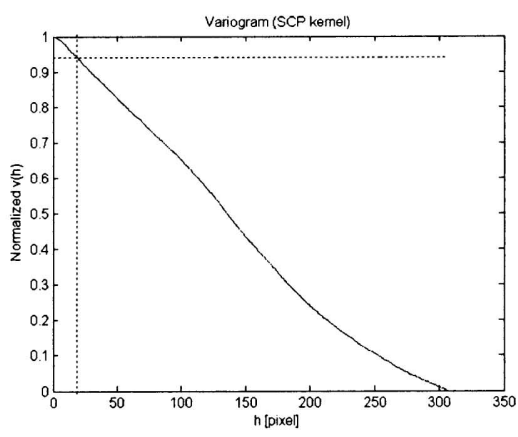

d

Fig. 4. SCP variogram for the tested stereopairs: a) Rubic, b) Phone, c) Fiesta, d) Street

Table 3. Comparison between results obtained with proposed method (SCP variogram) of the maximum disparity estimation and the human measure of the maximum disparity estimation

\begin{tabular}{|c|c|c|c|}
\hline Stereo pair & $\begin{array}{c}\text { Disparity range } \\
\text { estimated by human }\end{array}$ & SCP & Error \\
\hline Rubic & 11 & 11 & $0 \%$ \\
\hline Phone & 21 & 20 & $5 \%$ \\
\hline Fiesta & 18 & 15 & $20 \%$ \\
\hline Street & 17 & 19 & $12 \%$ \\
\hline Trees & 8 & 7 & $14 \%$ \\
\hline
\end{tabular}

\section{Conclusions}

A new method of the automatic estimation of the maximum disparity range with the variogram was invented. In this paper we found correlation between local minima of 
the variogram and value of the maximum disparity range. This method provides also a novel way of the variogram definition for the stereo images. The definition of the variogram kernel was also presented. In next stage of experiments the variogram kernel was modified due to its similarity to the measures used in the area-based stereo methods. New results obtained with modified SPC kernels allow to decrease estimation range error. All benefits make this method very attractive as an automatic estimation of a threshold parameter for almost any stereo-method.

Finally, an analysis of the methods was presented that showed usefulness of the presented concepts.

\section{References}

1. Alvarez, L., Deriche, R., Sanchez, J., Weickert, J.: Dense Disparity Map Estimation Respecting Image Discontinuities: A PDE and Scale-Space Based Approach. INRIA Technical Report No 3874 (2000)

2. Cyganek, B.: Computer Processing of 3D Images (in Polish), Exit (2002)

3. Cyganek, B., Borgosz, J.: A Comparative Study of Performance and Implementation of Some Area-Based Stereo Algorithms, CAIP'2001, Warsaw, Poland (2001) 709-716

4. Glass, C., Carr, J., Yang, H.M., Myers, D.: Application of spatial statistics to analyzing multiple remote sensing data set, Geotechnical Applications of Remote Sensing and Remote Data Transmission, American Society for Testing and Materials, Philadelphia, (1987) 139-150

5. Ramstein, G., Raffy, M.: Analysis of the Structure of Radiometric Remotely - Sensed Images, International Journal Of Remote Snesing, 10 (1989) 1049-1073

6. Scharstein, D.: View Synthesis Using Stereo Vision. Lecture Notes in Computer Science 1582, Springer-Verlag (1999)

7. Sporring, J., Nielsen, M., Florack, L., Johansen, P.: Gaussian Scale-Space Theory. Kluwer Academic Publishers (1997)

8. Starck, J.-L., Murtagh, F., Bijaoui, A.: Image Processing and Data Analysis. The Multiscale Approach. Cambridge University Press (2000) 